\title{
Retaining Large and Adjustable Elastic Strains of Kilogram-Scale Nb Nanowires
}

\author{
Shijie Hao, ${ }^{\dagger}$ Lishan Cui, ${ }^{* \dagger}$ Hua Wang,, Daqiang Jiang, ${ }^{\dagger}$ Yinong Liu, ${ }^{\S}$ Jiaqiang Yan, ${ }^{\|, \perp}$ Yang \\ Ren," ${ }^{*}$ Xiaodong Han, ${ }^{\nabla}$ Dennis. E. Brown, ${ }^{\circ}$ and Ju Li ${ }^{*}+$, !! \\ †State Key Laboratory of Heavy Oil Processing, China University of Petroleum, Beijing 102249, China \\ ${ }^{\ddagger}$ State Key Laboratory for Mechanical Behavior of Materials and Frontier Institute of Science and Technology, \\ Xi'an Jiaotong University, Xi’an 710049, China \\ ${ }^{\S}$ School of Mechanical and Chemical Engineering, The University of Western Australia, Crawley, WA 6009, \\ Australia \\ "Materials Science and Technology Division, Oak Ridge National Laboratory, Oak Ridge, TN 37831, USA \\ ${ }^{\perp}$ Department of Materials Science and Engineering, University of Tennessee, Knoxville, TN 37996, USA \\ ${ }^{\#}$ X-ray Science Division, Argonne National Laboratory, Argonne, Illinois 60439, USA \\ ${ }^{\nabla}$ Institute of Microstructure and Properties of Advanced Materials, Beijing University of Technology, Beijing \\ 100124, China
}

${ }^{\circ}$ Department of Physics, Northern Illinois University, De Kalb, Illinois 60115, USA

"Department of Nuclear Science and Engineering and Department of Materials Science and Engineering, Massachusetts Institute of Technology, Cambridge, Massachusetts 02139, USA

\begin{abstract}
:
Individual metallic nanowires can sustain ultra-large elastic strains of 4-7\%. However, achieving and retaining elastic strains of such magnitude in kilogram-scale nanowires are challenging. Here, we find that under active load, $\sim 5.6 \%$ elastic strain can be achieved in Nb nanowires embedded in a metallic matrix deforming by detwinning. Moreover, large tensile (2.8\%) and compressive $(-2.4 \%)$ elastic strains can be retained in kilogram-scale $\mathrm{Nb}$ nanowires when the external load was fully removed, and adjustable in magnitude by processing control. It is then demonstrated that the retained tensile elastic strains of $\mathrm{Nb}$ nanowires can increase their superconducting transition temperature and critical magnetic field, in comparison with the unstrained original material. This study opens new avenues for retaining large and tunable elastic strains in great quantities of nanowires and elastic-strain-engineering at industrial scale.
\end{abstract}

KEYWORDS: Nanowires, Shape memory alloy, High-energy X-ray diffraction, Elastic strain, Elastic strain engineering 
Crystals at ultrahigh levels of elastic strain may exhibit exceptional physical and chemical properties. ${ }^{1-8}$ A commercially successful example is the 'strained silicon technology', ${ }^{2,4}$ where a tensile elastic strain of a few percent is able to enhance the charge carrier mobility several times, which increases the CPU speed so significantly that it has delayed the breakdown of the Moore's law. However, when attempting to apply elastic strain engineering to energy technologies, one finds that in contrast to information technologies applications where the total mass of active material is tiny (the total weight of strained silicon in a CPU is $\sim 10^{-8} \mathrm{~kg}$ ), the total mass of active material to be strain-engineered for energy storage or power transmission has to be much larger. Consider superconducting cables as an example: if we cannot make kilogram- and kilometerscale strain-engineered superconductor, then elastic strain engineering will not have much real impact.

Much effort has been made to achieve and retain large elastic strains of crystal materials. ${ }^{4,7-}$ ${ }^{14}$ By lattice mismatch between films and substrates, large elastic strains can be retained in films without external load, but it is only applicable to films. ${ }^{4,-10}$ Near-crack-tip regions in crystal materials can bear large elastic strains, but their volume fractions are too small for meaningful application. ${ }^{11,12}$ Under high hydrostatic pressure, large elastic strains can be produced in gramscale matters, ${ }^{13},{ }^{14}$ but it cannot be retained without external load. To date, achieving and retaining large elastic strains of kilogram-scale crystal materials without external load are challenging.

Individual metallic nanowires have ultra-large elastic strains, often of the magnitude of 4$7 \% .^{2,11,15-17}$ Due to the limitation of using nanowires in their original forms in real applications, it has been conceived to create bulk composites with these nanowires as fillers. However, the ultra-large elastic strains of nanowires cannot be exploited in conventional composites with the matrices deforming by dislocation slip, ${ }^{18-20}$ as dislocations in the matrix slipping to the matrix/nanowire interface cause the atomic-level inelastic shear strain between two adjacent atomic planes exceeding $100 \%(b / d$, where $b$ is Burgers vector and $d$ is interplanar distance, is usually $>100 \%$ ) at the interface, ${ }^{21}$ which easily triggers plasticity in the nanowires. Inspired by this, we hypothesize that the matrix should not deform via sharp microscopic defects such as dislocations or cracks to achieve the ultra-large elastic strain of nanowires in a composite. ${ }^{22}$ To our knowledge, the shape memory alloy (SMA) in martensite state deforms by detwinning (variant reorientation) that is a collective shearing process of atoms with an atomic-level strain of $\sim 10 \%$, and is thus less likely to trigger plasticity in nanowires when the detwinning deformation front of the SMA matrix hits the nanowires. Moreover, the strain reversal of the SMA matrix is smaller than $2 \%$ upon tensile unloading which is much smaller than that $(4-7 \%)$ of the nanowires, which provide an opportunity for the ultra-large elastic strains of nanowires to be achieved firstly, and later retained in the composite after unloading, in the free-standing condition. This new design paradigm of free-standing composite is illustrated in Figure S1.

To verify the design, we developed an in situ composite wire (Figure 1a) composed of $\mathrm{Nb}$ nanowires and martensitic NiTi SMA matrix, which is fabricated by conventional hypo-eutectic ingot casting and wire drawing. Typical microstructure of the composite is shown in Figures 1b1d. The ribbon-shaped $\mathrm{Nb}$ nanowires, $4-20 \mathrm{~nm}$ in thickness and $20-200 \mathrm{~nm}$ in width, are well dispersed and well aligned along the axial direction of the composite wire. The $\mathrm{Nb}$ nanowires have lengths ranging from $1-100 \mu \mathrm{m}$ and a mean aspect ratio exceeding 100 . The NiTi matrix is 
in martensitic state at room temperature with twinned substructure. The volume fraction of the $\mathrm{Nb}$ nanowires is about $12 \%$. Chemical analysis of the composite is shown in Figure S2. The high-energy X-ray diffraction pattern of the composite (Figure 1e) can be fully indexed to bodycentered cubic Nb and B19'-NiTi phases. Figure 1f shows the pole figure of $\langle 110\rangle_{\mathrm{Nb}}$ in the wire axial direction. It is evident that the $\mathrm{Nb}$ nanowires are strongly oriented with [110] direction parallel to the wire axial direction.
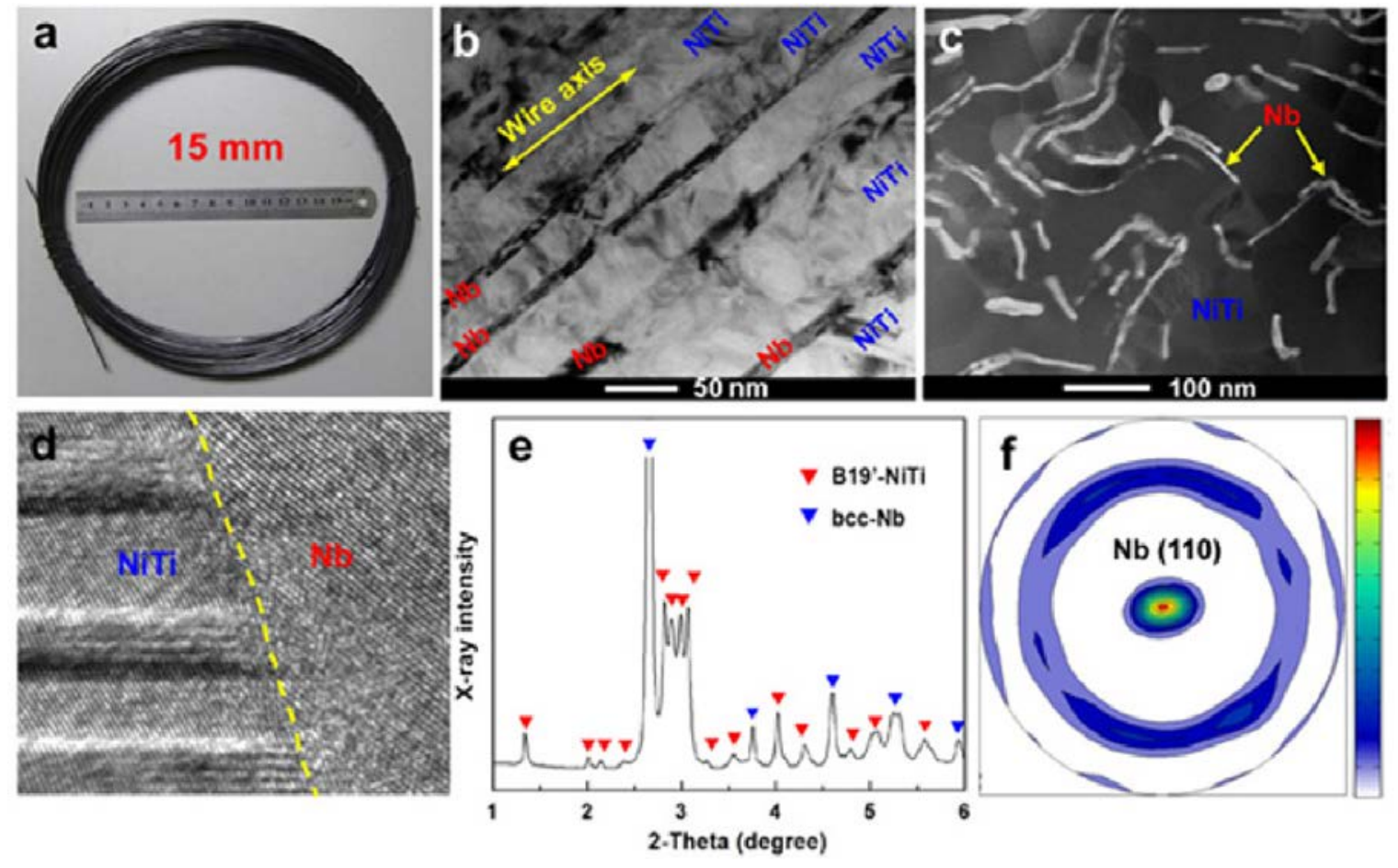

Figure 1. Microstructure of the composite wire. (a) A coil of the composite wire. (b) Brightfield TEM micrograph of a longitudinal-section of the composite wire. (c) Scanning TEM micrograph of a cross-section of the composite wire. (d) High-resolution TEM image of the interface between $\mathrm{Nb}$ nanowires and martensitic NiTi matrix with twin substructure. (e) 1D highenergy X-ray diffraction pattern of the composite wire. (f) $\mathrm{Nb}$-[110] pole figure in the wire axial direction.

To measure the elastic strains of the embedded $\mathrm{Nb}$ nanowires in the martensitic $\mathrm{NiTi}$ matrix, in situ synchrotron X-ray diffraction was carried out on the composite during tensile loading (inset of Figure 2a). Figure 2b shows a 2D high-energy X-ray diffraction pattern taken at a certain applied strain (1\%). Figure 2c shows the evolution of the X-ray diffraction intensity of B19'-NiTi (001) planes along the Debye-Scherrer ring (as indicated in Figure 2b) recorded at different applied strains. It is seen that the diffraction intensity of B19'-NiTi (001) planes along the Debye-Scherrer ring concentrates approximately equally at 6 locations, separated by $\sim 60^{\circ}$. Upon loading, the B19'-NiTi (001) diffraction intensity changes significantly to four dominant orientations, indicating that the martensitic NiTi matrix has undergone detwinning (variant reorientation) during deformation. ${ }^{23}$ Figures $2 \mathrm{~d}-2 \mathrm{e}$ show transmission electron microscopy images of the martensitic NiTi matrix before and after a tensile deformation of $8.7 \%$ respectively. These further demonstrate that the martensitic NiTi matrix has undergone detwinning during the deformation. Figure 2a shows the evolution of $d$-spacing strain with 
respect to applied strain for the $\mathrm{Nb}$ (110) planes perpendicular to the loading direction. It is seen that the embedded $\mathrm{Nb}$ nanowires reached a maximum tensile elastic strain of $5.6 \%$ (at $7.5 \%$ applied strain). The elastic strain was induced by the deformation of SMA matrix through detwinning. It is considered that the stress induced at interface is shear stress which resulted in tensile strain in the (110) direction of the nanowire. However, the specific strain transfer mechanism between the martensitic NiTi SMA matrix and the Nb nanowire during tensile loading is still not very clear. This large elastic strain is comparable to those of freestanding nanowires and much larger than those of the nanowires embedded in metal matrices deforming by dislocation slip ( 1.5\%) (Figure S3). ${ }^{19}, 20$
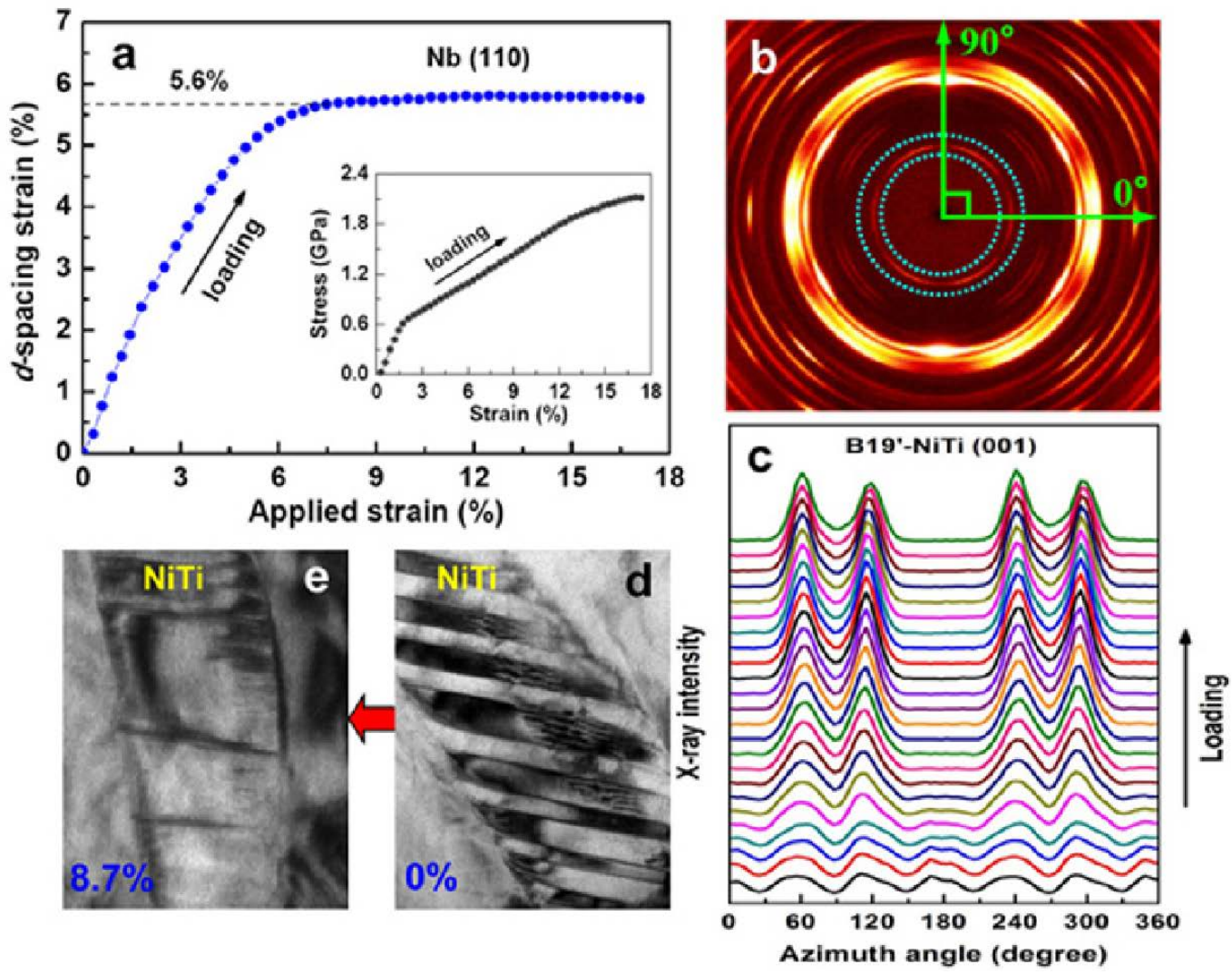

Figure 2. Achieving ultra-large elastic strain of Nb nanowires in martensitic NiTi matrix. (a) Evolution of $d$-spacing strain with respect to applied strain for the $\mathrm{Nb}$ (110) plane perpendicular to the wire axial direction during tensile loading. The inset is the macroscopic stress-strain curve of the composite. (b) 2D high-energy X-ray diffraction pattern of the composite at a certain tensile strain level (1\%). (c) Evolution of X-ray diffraction intensity of B19'-NiTi <001> planes versus the azimuth angle (as determined along the Debye-Scherrer ring indicated in (b)) during tensile loading. (d-e) TEM micrographs of twin morphologies of the martensitic NiTi matrix before and after a tensile deformation cycle to $8.7 \%$.

To determine that the large tensile elastic strain of the $\mathrm{Nb}$ nanowires can be retained in the composite after unloading, in situ synchrotron X-ray diffraction was performed on the composite through multiple-step tensile deformation cycles, as shown in Figure 3a. Evolution of X-ray diffraction peaks for $\mathrm{Nb}(110)$ and B19'-NiTi (001) planes through the deformation cycles is shown in Figure 3b. Evolution of $d$-spacing strain with respect to applied strain for the Nb (110) 
plane perpendicular to the loading direction is shown in Figure 3c. It is observed that tensile elastic strains of $0.4 \%, 1.1 \%, 1.4 \%, 2.3 \%$ and $2.8 \%$ in the $\mathrm{Nb}$ nanowires were retained after deformations to $1.5 \%, 3.0 \%, 4.5 \%, 6.2 \%$ and $8.7 \%$, respectively. The dependence of the retained tensile elastic strains in the $\mathrm{Nb}$ nanowires on the prior tensile cycle strains of the composite is given in Figure S4. This demonstrates that the magnitude of retained tensile elastic strain in the $\mathrm{Nb}$ nanowires can be tailored by adjusting the prior tensile cycle strain of the composite. Moreover, these retained tensile elastic strains (up to 2.8\%) in the Nb nanowires are larger than those achieved in thin films on substrates reported in the literature (Figure S5). ${ }^{24-29}$

The retention of tensile elastic stains in the Nb nanowires after unloading can be understood as following (Figure S1). Upon loading to a tensile strain of $\varepsilon_{t}$, the martensitic NiTi matrix undergoes a small elastic deformation (O-E) followed by a large detwinning (variant reorientation) deformation (E-F), while the $\mathrm{Nb}$ nanowires undergo a large elastic deformation (O-A) followed by a small plastic deformation (A-B). Upon unloading, both the martensitic NiTi matrix and the $\mathrm{Nb}$ nanowires recover elastically. However, because the elastic strain of the martensitic NiTi matrix ( $1 \%$, Figure S6) is much smaller than that of the Nb nanowires ( $5.6 \%$, Figure 2a), the NiTi matrix hinders the full recovery of the Nb nanowires, and the composite equilibrates at point $\mathrm{C}$ (Figure S1), causing a large tensile elastic strain in the Nb nanowires ( $\varepsilon N W-e)$ and a compressive elastic strain in the NiTi matrix (Figure S6) at the end of the unloading.

Large retained compressive elastic strains can also be created in the $\mathrm{Nb}$ nanowires by heating the pre-deformed composite. Figure 3d shows the evolution of the retained elastic strain of the $\mathrm{Nb}$ nanowires in a composite sample pre-deformed to $8.7 \%$ in tension (Figure S7) upon heating. It is seen that the retained tensile elastic strain in the $\mathrm{Nb}$ nanowires was released rapidly accompanying the reverse phase transformation $\left(\mathrm{B} 19^{\prime} \rightarrow \mathrm{B} 2\right)$ of the NiTi matrix (Figure S8). After heating, a large compressive elastic strain of $-2.4 \%$ was retained in the $\mathrm{Nb}$ nanowires. This retained compressive elastic strain in the Nb nanowires is comparable to those reported for thin films on substrates and embedded nanoinclusions in films, ${ }^{24-29}$ as shown in Figure S5. This can be understood as following. Upon heating, the martensitic NiTi matrix returned to its initial predeformation shape by the reverse phase transformation $\left(\mathrm{B} 19^{\prime} \rightarrow \mathrm{B} 2\right)$ (Figure S8). Because the Nb nanowires underwent a plastic deformation (A-B in Figure S7-b) during the pre-deformation, the plastically deformed $\mathrm{Nb}$ nanowires hindered the full shape recovery of the NiTi matrix upon heating, resulting in a large compressive elastic strain in the $\mathrm{Nb}$ nanowires and a tensile elastic strain in the NiTi matrix. 

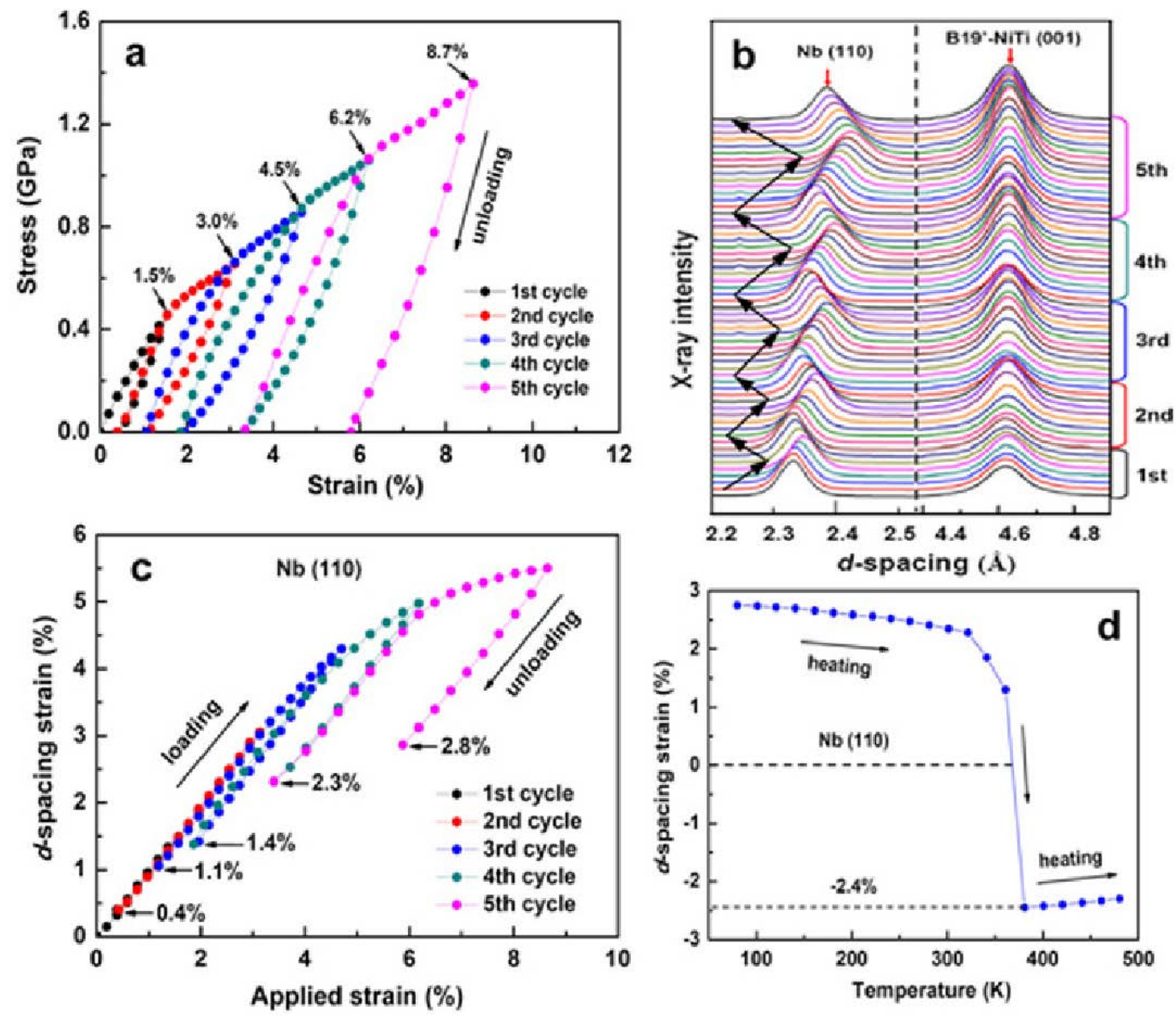

Figure 3. Retaining large elastic strains of great quantity Nb nanowires without external load. (a) Stress-strain curves of multiple-step tensile deformation cycles of the composite. (b) Evolution of high-energy X-ray diffraction peaks for Nb (110) and B19' - NiTi (001) planes through the multiple-step tensile cycles. (c) Evolution of $d$-spacing strain with respect to applied strain for the $\mathrm{Nb}$ (110) plane perpendicular to the wire axial direction during the multiple-step tensile cycles. (d) Evolution of $d$-spacing strain with respect to temperature for the Nb (110) plane perpendicular to the wire axial direction in the composite pre-deformed to $8.7 \%$ in tension.

Niobium (Nb) is a low-temperature superconductor. ${ }^{30}$ We herein measured the effect of the retained elastic strains on the superconducting properties of Nb nanowires. Figures S9 and S10 show the superconducting transition curves and the magnetization versus magnetic field $(\mathrm{M}-\mathrm{H})$ curves of the $\mathrm{Nb}$ nanowires subjected to different retained tensile elastic strains, from $\varepsilon_{[110]}=0$ to $\varepsilon[110]=2.6 \%$. Dependences of the superconducting transition temperature $\left(T_{\mathrm{c}}\right)$ and the critical magnetic fields $\left(H_{\mathrm{c}-1}\right.$ and $\left.H_{\mathrm{c}-2}\right)$ on the retained tensile elastic strains are shown in Figures $4 \mathrm{a}$ and $4 \mathrm{~b}$, respectively. It is seen that the $T_{c}, H_{c-1}$ and $H_{c-2}$ of the $\mathrm{Nb}$ nanowires significantly increased with increasing the retained tensile elastic strains, demonstrating that the retained tensile elastic strains improve the superconducting properties of great quantities Nb nanowires. 

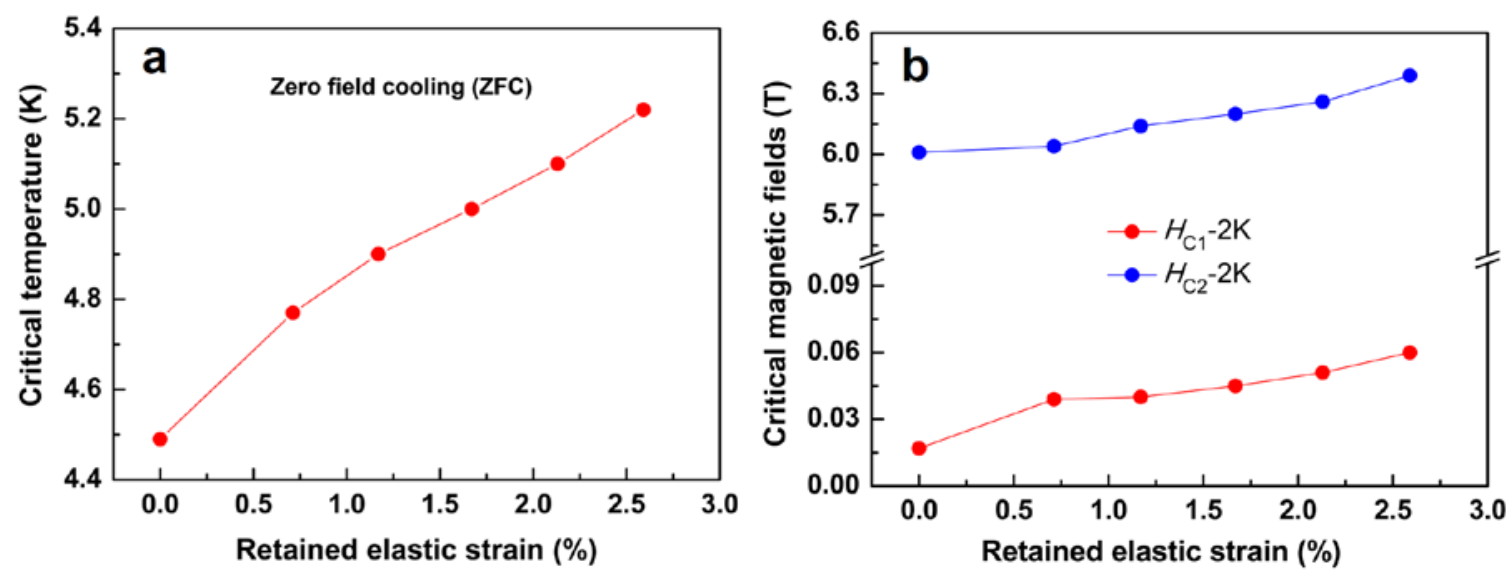

Figure 4. Effect of retained tensile elastic strain on superconducting properties of great quantity Nb nanowires. (a) Dependence of the superconducting transition temperature $\left(T_{\mathrm{c}}\right.$, midpoint of the transition curve) of $\mathrm{Nb}$ nanowires on the retained tensile elastic strains. (b) Dependence of the critical magnetic fields (the lower critical field $H_{\mathrm{c}-1}$ and the upper critical field $H_{\mathrm{c}-2}$ ) of $\mathrm{Nb}$ nanowires on the retained tensile elastic strains.

In summary, the ultra-large elastic straining capability of $\mathrm{Nb}$ nanowires were exploited in a metal matrix deforming by detwinning. Both the large tensile and compressive elastic strains of $\mathrm{Nb}$ nanowires were retained in the free-standing composite after thermal-mechanical treatments, and the retained elastic strains can also be adjusted in magnitude. It is demonstrated that the retained tensile elastic strains can increase the $T_{\mathrm{c}}, H_{\mathrm{c}-1}$ and $H_{\mathrm{c}-2}$ of great quantities of $\mathrm{Nb}$ nanowires. This paradigm of locking in large elastic strains in large quantity nanowires in a freestanding composite opens new avenues for retaining large and tunable elastic strains of kilogram-scale crystal materials and exploiting “elastic strain engineering” at industrial scale.

\section{Supporting Information}

Additional information and figure as noted in the text. This material is available free of charge via the Internet at http://pubs.acs.org

\section{Corresponding Authors}

*E-email: lishancui63@126.com,*E-email: ren@aps.anl.gov, *E-email: liju@mit.edu

\section{Notes}

The authors declare no competing financial interest

\section{ACKNOWLEDGEMENTS}

We thank X. Shi and Z. Liu for help in the TEM observations. This work was supported by the Key Program of the NSFC (51231008), the National 973 program of China (2012CB619400), the ARC grant (DP140103805), the NSFC (51471187 and 11474362), Beijing Natural Science Foundation (2152026), and the Science Foundation of China University of Petroleum, Beijing (2462013YJRC005). The use of the Advanced Photon Source was supported by the US Department of Energy, Office of Science, and Office of Basic Energy Science under Contract No. DE-AC02-06CH11357. J.Yan acknowledges support from the US Department of Energy, Office of Science, Basic Energy Sciences, Materials Sciences and Engineering Division. 


\section{REFERENCES}

(1) Li, J.; Shan, Z. W.; Ma, E. Elastic Strain Engineering for Unprecedented Materials Properties. Mrs Bull. 2014, 39, 108-117.

(2) Zhu, T.; Li, J. Ultra-Strength Materials. Prog. Mater Sci. 2010, 55, 710-757.

(3) Hicks, C. W.; Brodsky, D. O.; Yelland, E. A.; Gibbs, A. S.; Bruin, J. A. N.; Barber, M. E.; Edkins, S. D.; Nishimura, K.; Yonezawa, S.; Maeno, Y.; Mackenzie, A. P. Strong Increase of Tc of $\mathrm{Sr}_{2} \mathrm{RuO}_{4}$ Under Both Tensile and Compressive Strain. Science 2014, 344, 283-285.

(4) Jacobsen, R. S.; Andersen, K. N.; Borel, P. I.; Fage-Pedersen, J.; Frandsen, L. H.; Hansen, O.; Kristensen, M.; Lavrinenko, A. V.; Moulin, G.; Ou, H.; Peucheret, C.; Zsigri, B.; Bjarklev, A. Strained Silicon as a New Electro-Optic Material. Nature 2006, 441, 199-202.

(5) Levy, N.; Burke, S. A.; Meaker, K. L.; Panlasigui, M.; Zettl, A.; Guinea, F.; Neto, A. H. C.; Crommie, M. F. Strain-Induced Pseudo-Magnetic Fields Greater Than 300 Tesla in Graphene Nanobubbles. Science 2010, 329, 544-547.

(6) Cao, J.; Ertekin, E.; Srinivasan, V.; Fan, W.; Huang, S.; Zheng, H.; Yim, J. W. L.; Khanal, D. R.; Ogletree, D. F.; Grossmanan, J. C.; Wu, J. Strain Engineering and One-Dimensional Organization of Metal-Insulator Domains in Single-Crystal Vanadium Dioxide Beams. Nat. Nanotechnol. 2009, 4, 732-737.

(7) Strasser, P.; Koh, S.; Anniyev, T.; Greeley, J.; More, K.; Yu, C. F.; Liu, Z. C.; Kaya, S.; Nordlund, D.; Ogasawara, H.; Toney, M. F.; Nilsson, A. Lattice-Strain Control of the Activity in Dealloyed Core-Shell Fuel Cell Catalysts. Nat. Chem. 2010, 2, 454-460.

(8) Schlom, D. G.; Chen, L. Q.; Eom, C. B.; Rabe, K. M.; Streiffer, S. K.; Triscone, J. M. Strain Tuning of Ferroelectric Thin Films. Annu. Rev. Mater. Res. 2007, 37, 589-626.

(9) MaCmanus-Driscoll, J. L.; Zerrer, P.; Wang, H. Y.; Yang, H.; Yoon, J.; Fouchet, A.; Yu, R.; Blamire, M. G.; Jia, Q. X. Strain Control and Spontaneous Phase Ordering in Vertical Nanocomposite Heteroepitaxial Thin Films. Nat. Mater. 2008, 7, 314-320.

(10) Jang, H. W.; Baek, S. H.; Ortiz, D.; Folkman, C. M.; Das, R. R.; Chu, Y. H.; Shafer, P.; Zhang, J. X.; Choudhury, S.; Vaithyanathan, V.; Chen, Y. B.; Felker, D. A.; Biegalski, M. D.; Rzchowski, M. S.; Pan, X. Q.; Schlom, D. G.; Chen, L. Q.; Ramesh, R.; Eom, C. B. StrainInduced Polarization Rotation in Epitaxial (001) $\mathrm{BiFeO}_{3}$ Thin Films. Phys. Rev. Lett. 2008, 101, 107602.

(11) Yue, Y. H.; Liu, P.; Zhang, Z.; Han, X. D.; Ma, E. Approaching the Theoretical Elastic Strain Limit in Copper Nanowires. Nano Lett. 2011, 11, 3151-3155.

(12) Rice, J. The Elastic-Plastic Mechanics of Crack Extension. Int. J. Fract. 1968, 4, 41-47.

(13) Duffy, T. S.; Shen, G.; Heinz, D. L.; Shu, J.; Ma, Y.; Mao, H.-K.; Hemley, R. J.; Singh, A. K. Lattice Strains in Gold and Rhenium under Nonhydrostatic Compression to $37 \mathrm{GPa}$. Phys. Rev. B 1999, 60, 15063-15073.

(14) Singh, A. K.; Balasingh, C.; Mao, H.-k.; Hemley, R. J.; Shu, J. Analysis of Lattice Strains Measured under Nonhydrostatic Pressure. J. Appl. Phys. 1998, 83, 7567-7575.

(15) Richter, G.; Hillerich, K.; Gianola, D. S.; Monig, R.; Kraft, O.; Volkert, C. A. Ultrahigh Strength Single Crystalline Nanowhiskers Grown by Physical Vapor Deposition. Nano Lett. 2009, 9, 3048-3052.

(16) Wong, E. W.; Sheehan, P. E.; Lieber, C. M. Nanobeam Mechanics: Elasticity, Strength and Toughness of Nanorods and Nanotubes. Science 1997, 277, 1971-1975.

(17) Tian, L.; Cheng, Y. Q.; Shan, Z. W.; Li, J.; Wang, C. C.; Han, X. D.; Sun, J.; Ma, E. Approaching the Ideal Elastic Limit of Metallic Glasses. Nat. Commun. 2012, 3,609. 
(18) Dzenis, Y. Structural Nanocomposites. Science 2008, 319, 419-420.

(19) Vidal, V.; Thilly, L.; Van Petegem, S.; Stuhr, U.; Lecouturier, F.; Renault, P. O.; Van Swygenhoven, H. Plasticity of Nanostructured Cu-Nb-based Wires: Strengthening Mechanisms Revealed by in Situ Deformation under Neutrons. Scripta Mater. 2009, 60, 171-174.

(20) Scheuerlein, C.; Stuhr, U.; Thilly, L. In-Situ Neutron Diffraction under Tensile Loading of Powder-in-Tube $\mathrm{Cu} / \mathrm{Nb}_{3} \mathrm{Sn}$ Composite Wires: Effect of Reaction Heat Treatment on Texture, Internal Stress State, and Load Transfer. Appl. Phys. Lett. 2007, 91, 042503.

(21) Ogata, S.; Li, J.; Yip, S. Ideal Pure Shear Strength of Aluminum and Copper. Science 2002, 298, 807-811.

(22) Hao, S. J.; Cui, L. S.; Jiang, D. Q.; Han, X. D.; Ren, Y.; Jiang, J.; Liu, Y. N.; Liu, Z. Y.; Mao, S. C.; Wang, Y. D.; Li, Y.; Ren, X. B.; Ding, X. D.; Wang, S.; Yu, C.; Shi, X. B.; Du, M. S.; Yang, F.; Zheng, Y. J.; Zhang, Z.; Li, X. D.; Brown, D. E.; Li, J. A Transforming Metal Nanocomposite with Large Elastic Strain, Low Modulus, and High Strength. Science 2013, 339, 1191-1194.

(23) Otsuka, K.; Ren, X. Physical Metallurgy of Ti-Ni-Based Shape Memory Alloys. Prog. Mater. Sci. 2005, 50, 511-678.

(24) Cai, Z. H.; Kuru, Y.; Han, J. W.; Chen, Y.; Yildiz, B. Surface Electronic Structure Transitions at High Temperature on Perovskite Oxides: The Case of Strained $\mathrm{La}_{0.8} \mathrm{Sr}_{0.2} \mathrm{CoO}_{3}$ Thin Films. J. Am. Chem. Soc. 2011, 133, 17696-17704.

(25) Hobart, K. D.; Kub, F. J.; Fatemi, M.; Twigg, M. E.; Thompson, P. E.; Kuan, T. S.; Inoki, C. K. Compliant Substrates: A Comparative Study of the Relaxation Mechanisms of Strained Films Bonded to High and Low Viscosity Oxides. J. Electron. Mater. 2000, 29, 897-900.

(26) Zeches, R. J.; Rossell, M. D.; Zhang, J. X.; Hatt, A. J.; He, Q.; Yang, C. H.; Kumar, A.; Wang, C. H.; Melville, A.; Adamo, C.; Sheng, G.; Chu, Y. H.; Ihlefeld, J. F.; Erni, R.; Ederer, C.; Gopalan, V.; Chen, L. Q.; Schlom, D. G.; Spaldin, N. A.; Martin, L. W.; Ramesh, R. A StrainDriven Morphotropic Phase Boundary in $\mathrm{BiFeO}_{3}$. Science 2009, 326, 977-980.

(27) Zhang, K. H. L.; Lazarov, V. K.; Veal, T. D.; Oropeza, F. E.; McConville, C. F.; Egdell, R. G.; Walsh, A. Thickness Dependence of the Strain, Band Gap and Transport Properties of Epitaxial $\mathrm{In}_{2} \mathrm{O}_{3}$ Thin Films Grown on Y-Stabilised $\mathrm{ZrO}_{2}$ (111). J. Phys-Condens. Mat. 2011, 23, 334211.

(28) Infante, I. C.; Sanchez, F.; Fontcuberta, J.; Wojcik, M.; Jedryka, E.; Estrade, S.; Peiro, F.; Arbiol, J.; Laukhin, V.; Espinos, J. P. Elastic and Orbital Effects on Thickness-Dependent Properties of Manganite Thin Films. Phys. Rev. B 2007, 76, 224415.

(29) Horide, T.; Kitamura, T.; Ichinose, A.; Matsumoto, K. Elastic Strain Evolution in Nanocomposite Structure of $\mathrm{YBa}_{2} \mathrm{Cu}_{3} \mathrm{O}_{7}+\mathrm{BaZrO}_{3}$ Superconducting Films. Jpn. J. Appl. Phys. 2014, 53, 083101.

(30) Laverick, C. TMS Symposium on Niobium and Niobium Alloys in Superconducting Applications Niobium demand and superconductor applications: An overview. J. Less-Common Met. 1988, 139, 107-122. 
TOC Graphic
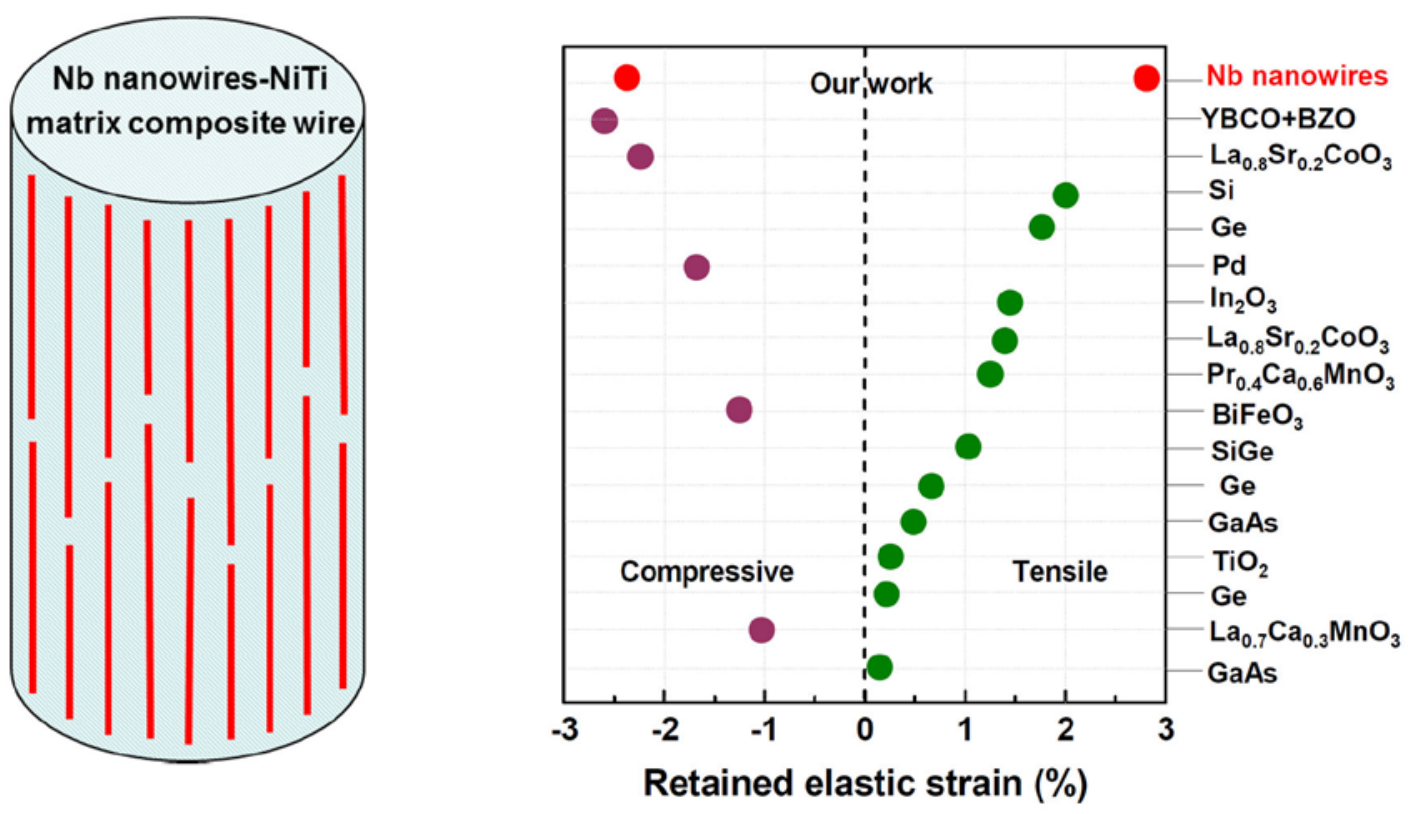